\title{
FUE vs. FUT-MD: Study of 1,780 Follicles in Four Patients
}

\author{
Michael L. Beehner, MD, FISHRS Saratoga Springs, New York USA mlbeehner@saratogahair.com
}

\section{Introduction}

During the past ten years, there has been a tremendous increase in the use of "follicular unit extraction" (FUE) as a method for harvesting donor hair in hair transplant surgery. Amazingly, this has occurred despite the fact that there are virtually no published, detailed studies showing that the survival of FUE harvested follicles is favorably comparable to those obtained by a strip harvest with microscopic dissection (FUT-MD), which has been the "gold standard" for the past 25 years.

Most hair surgeons familiar with the FUE method agree that the "learning curve" for mastering manual FUE is steep and long in duration. Many of the physicians entering the field in recent years have been led to believe that knowing how to perform FUE will suffice to cover all situations in surgical hair restoration.

Because I felt there was an urgent need for a study comparing the two harvesting methods, I conducted a detailed study of four patients over a 3-year period. All four patients possessed "shiny bald" frontal areas upon which to conduct the study (Figures 1 and 2). In all, 890 FUE follicles were followed along with 890 FUT-MD follicles.

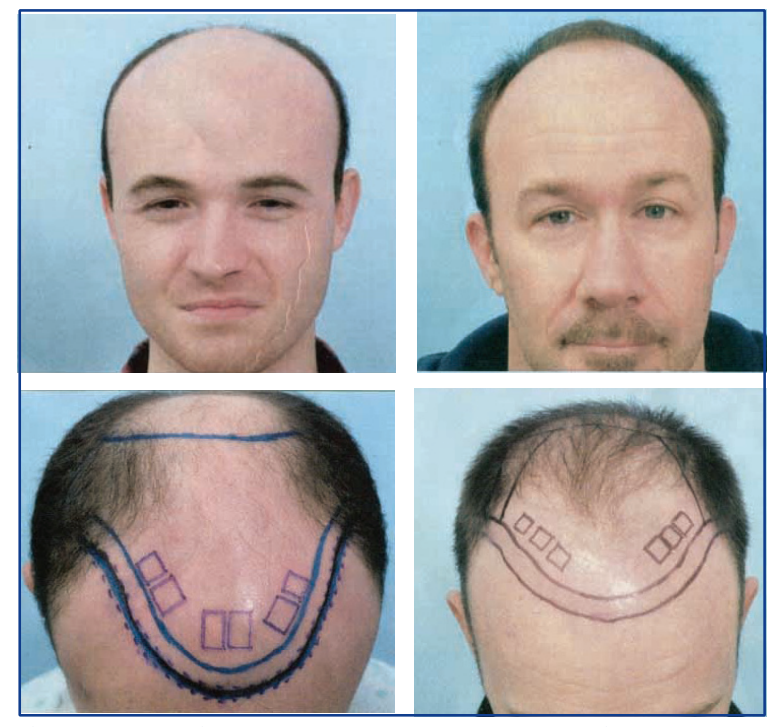

Figure 1. Study patients 1 (left) and 2 (right): top row, front view; bottom row,

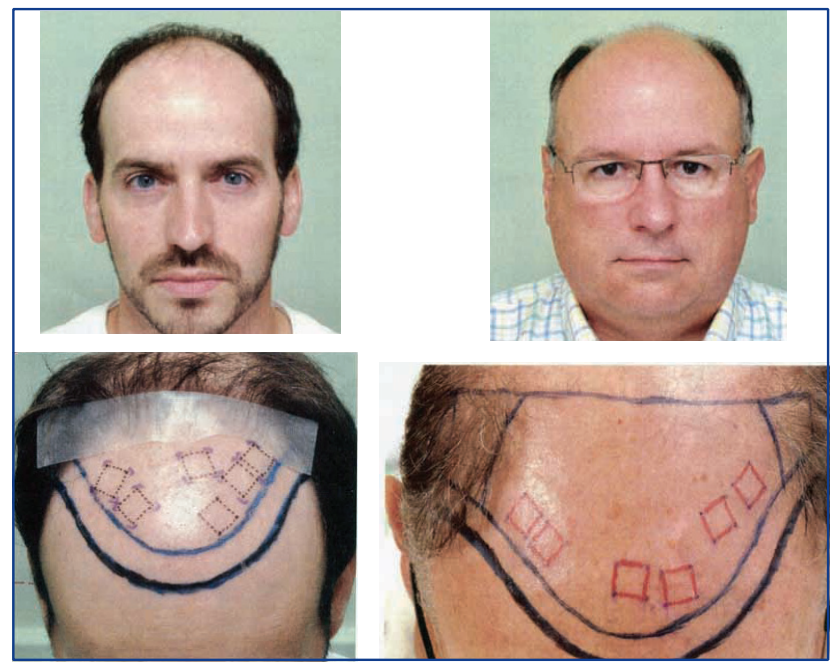

Figure 2. Study patients 3 (left) and 4 (right): top row, front view; bottom row, top view
To have a better perspective on our study, I feel it is important to define our surgical team's level of experience at the time we did this study. In late 2012 when we embarked on this research, I and my staff had conducted around 90 FUE surgeries over an 8-year period and felt quite comfortable using James Harris's SAFE II System. We first harvested FUE grafts using the 3-step manual Harris SAFE System method for 3 years, and then switched to the SAFE II System at that point. Most of our FUE procedures were in the 400-600 range as a supplement to a strip harvest or for obtaining grafts to camouflage a donor scar. I, as the physician, exclusively performed all of the tasks except for trimming and placing the grafts. Our maximum number of FUE grafts for one day had been 1,300 . I certainly would concede that some of the very experienced FUE practitioners who have mastered this technique over many years may very well have obtained higher follicle survival percentages than we did. On the other hand, I am quite sure our results were superior to those of the average physician in his or her first couple of years performing FUE. Regardless, this study was embarked upon in order to find out how the two donor harvesting methods stacked up against each other.

\section{Materials and Method}

The SAFE II System with the round, dull punch (both 0.9 and $1.0 \mathrm{~mm}$ diameter) was used for harvesting the FUE grafts. The donor strips were harvested using the classic "one blade" Limmer method, with slivering and the use of $10 \times$ stereoscopic microscopes. The 1-hair FUs were placed in $0.8 \mathrm{~mm}$ slit sites; 2-hair FUs in $1.0 \mathrm{~mm}$ slits, and 3-hair FUs in $1.1 \mathrm{~mm}$ sites. All slits were in parallel (sagittal) orientation or slightly oblique off of strict parallel. (See Figure 3.) All tissue and grafts, when out of the body, were kept moist and cool in Plasmalyte solution at $4^{\circ} \mathrm{Centi-}$ grade. Each study box measured $1.1 \mathrm{~cm}$ $\times 1.1 \mathrm{~cm}\left(1.21 \mathrm{~cm}^{2}\right.$ area), and a $2 \mathrm{~mm}$ wide bald "moat" was left around each box. Light brown tattoo dots were placed at the corners of each box for easy later identification Figure 4).

In our first two pa-

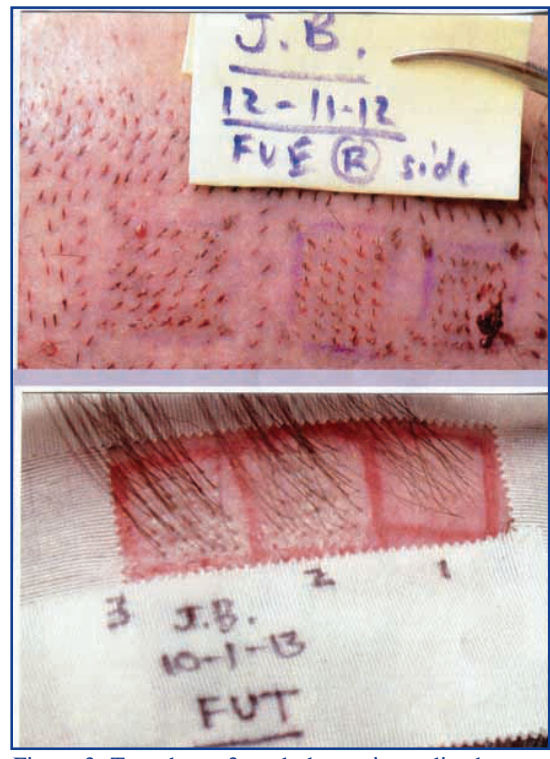

Figure 3. Top photo: 3 study boxes immediately postop; bottom photo: 3 FUE study boxes at 10 months in patient \#2 tients, we created paired boxes for 1-, 2-, and 3-hair grafts. Our third patient had too few natural 1-hair FU donor bundles, and our fourth patient had too few 3-hair grafts, so each of these patients had an extra box of 2-hair FUs to substitute for the missing size grafts.

For each patient, we first harvested the donor strip and sutured 
it together before harvesting the FUE grafts. The FUE grafts were harvested in the occipital region approximately $2 \mathrm{~cm}$ superior to the suture line. In each patient, the

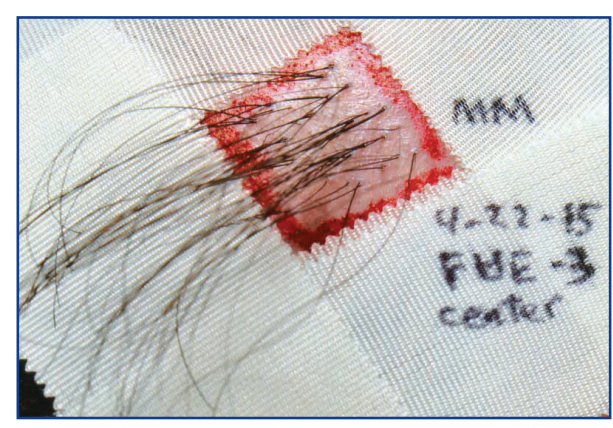
Figure 4. Single study box isolated and prepared for hair count. FUE grafts were placed first before placing the FUT-MD ones.

The first hair counts were performed at 14, 10, 11, and 14 months due to various times the 4 patients were able to return. All of the study grafts for the first two patients were "out of body" around 5-7 hours, which was longer than the 3-4 hour time period for the last two patients, when we made a concerted effort to shorten this interval. On average, for each patient the FUE grafts were out of body around one hour less than were the FUT-MD grafts.

The four patients ranged in age from 27 to 56, with the middle two patients in their mid-30s. None were smokers and none had been using finasteride or minoxidil. For the first two patients, the grafts were all placed by our head nurse ( 21 years of experience), and for the final two patients, all grafts were placed by me, using a "modified stick-and-place" method, in order to minimize the chance of trauma in the entry phase of planting.

\section{Results}

Table 1 below shows the individual results for each patient. Across the board, the FUT-MD grafts survived with higher percentages than the FUE ones. Notable was the fact that patient \#1 had very poor growth in all three of his FUE study boxes, and yet his FUT-MD grafts survived relatively well, comparable to the other three patients.

\begin{tabular}{|l|c|c|c|}
\hline PATIENT & FUE & FUT-MD & Difference \\
\hline Patient \#1 (E.B.) & $74 / 220(33.6 \%)$ & $183 / 220(83 \%)$ & $49.40 \%$ \\
\hline Patient \#2 (J.B.) & $163 / 220(74.1 \%)$ & $192 / 220(87.3 \%)$ & $13.20 \%$ \\
\hline Patient \#3 (M.M.) & $187 / 250(74.8 \%)$ & $238 / 250(95.2 \%)$ & $20.40 \%$ \\
\hline Patient \#4 (P.K.) & $123 / 200(61.5 \%)$ & $152 / 200(76 \%)$ & $14.50 \%$ \\
\hline \multicolumn{4}{|c}{ Table 1. Individual Patient Comparisons } \\
\hline
\end{tabular}

The oldest patient, at age 56, had the overall poorest growth with $61.5 \%$ survival of FUE grafts and $76 \%$ for the FUT-MD follicles. Patient \#3 (35 years of age) had the best overall growth with $74.8 \%$ for FUE and $95.2 \%$ for FUT-MD.

As shown in Table 2, if all 4 patients' results are bunched together, there was a $61.4 \%$ survival for the 890 FUE follicles and an $86 \%$ survival rate for the FUT-MD follicles. If patient \#1, the "outlier" with the $33 \%$ FUE survival, is dropped, the other three patients had a $70.1 \%$ survival rate of FUE follicles and $86.9 \%$ survival rate for FUT-MD ones.

\begin{tabular}{|c|ccc|}
\cline { 2 - 4 } \multicolumn{1}{c|}{} & FUE Grafts & FUT-MD Grafts & Difference \\
\hline All Four Patients Combined & $547 / 890(61.4 \%)$ & $765 / 890(86 \%)$ & $24.60 \%$ \\
\hline Three patients ("out-lier" removed) & $473 / 670(70.1 \%)$ & $582 / 670(86.9 \%)$ & $16.80 \%$ \\
\hline \multicolumn{2}{r}{ Table } & 2. Combined Results
\end{tabular}

Extremely vellous hairs were not counted. Hairs that were somewhat miniaturized were counted and numbered 48 among the FUT-MD grafts and 27 of the FUE grafts. There were 17 grafts that resulted in one additional hair growing beyond the number planted, and these extra hairs were not included in the results tabulated.
In looking at how 1-, 2-, and 3-hair FUs did respectively versus each other with the two harvesting methods, the FUE/FUT-MD results were $58 \% / 86 \%$ for 1 -hair grafts, $59 \% / 82 \%$ for 2 -hair grafts, and $66 \% / 91 \%$ for 3-hair grafts.

\section{Discussion}

Overall, there was a consistent increased survival percentage for the FUT-MD grafts in every pair of the study boxes, despite the fact that they were "out of body" at least 1 hour longer than the FUE grafts. In performing a detailed study like this, unfortunately, time quickly passes while you are carefully trying to perform and record each step properly, and as a result the grafts were out of the body for our first two patients longer than I would have wished, and certainly longer than would occur in most of our day-to-day surgeries.

I would stress the fact that we used great care in carefully placing all of the study grafts, especially the FUE ones, which are more difficult to place due to the loss of fatty surrounding tissue in most of the grafts. In trying to account for the lower survival of the FUE grafts, I would speculate that this tearing off of the surrounding subcutaneous tissue during the "pluck" phase of graft removal would be the prime suspect for this poorer growth (Figure 5). The increased difficulty in graft placement and the potential for desiccation

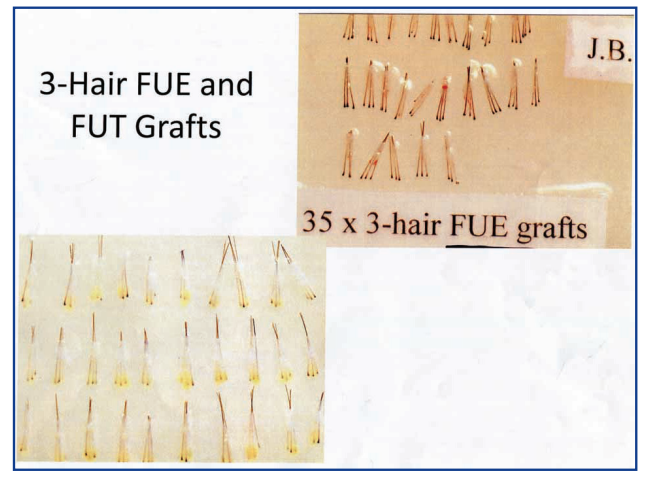

Figure 5. Study FUE and FUT grafts side-by-side could play a role in this occurrence.

It should be noted that all of the study boxes were positioned in the middle of a great many grafts, which extended over most of the balding scalp. Placing these boxes by themselves with surrounding "virgin" scalp would presumably have resulted in higher survival rates.

In time, there will certainly be additional studies comparing FUE and FUT-MD grafts. It will be interesting to see if future results are similar to mine or not. It will also be interesting to see if other investigators find occasional patients with extremely poor FUE growth as my first patient showed. I have no way of knowing whether this is a one in a hundred rare occurrence for FUE grafts in any given patient, or whether it will occur more frequently. If the former, then his results should be dropped from my study's conclusions, and my final results should show a 70.1\% survival for FUE grafts and an $86.9 \%$ survival for the FUT-MD ones. On the other hand, if it is a more than occasional occurrence, then my study should show that $61.4 \%$ of FUE grafts survive in the hands of a moderately competent surgeon and $86.9 \%$ for the FUT-MD grafts.

\section{Conclusion}

I have heard at least one FUE surgeon state that studies aren't necessary to prove anything. Manny Merritt showed many years ago that the human eye couldn't differentiate hair growing at $100 \%$ from that growing at only $50 \%$. I think our patients have a right to know if any of their donor hair is being wasted due to the harvesting method chosen. If there is a difference, the patient should be informed before choosing how to proceed. 